\title{
Descripción clínica y epidemiológica de pacientes con pancreatitis crónica en un hospital de alta complejidad en Cali, 2011 a 2017
}

\section{Clinical and epidemiological description of patients with chronic pancreatitis treated at a quaternary care hospital in Cali, 2011-2017}

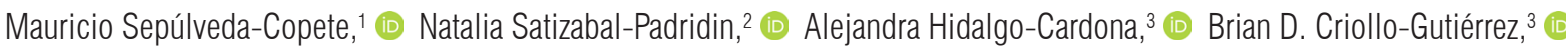
Angélica Tobón-Guevara, ${ }^{4}$ (D) Andrés M. Castro-Llanos, ${ }^{2}$ (1) Marisol Aguirre-Rojas, ${ }^{2}$ (C) Carlos A. Rojas-Rodríguez. ${ }^{1 *}$

\author{
GaCCESO ABIERTO \\ Citación: \\ Sepúlveda-Copete M, Satizabal-Padridin N, \\ Hidalgo-Cardona A, Criollo-Gutiérrez B \\ Tobón-Guevara A, Castro-Llanos A, Aguirre- \\ Rojas M, Rojas-Rodríguez C. Descripción \\ clínica y epidemiológica de pacientes con \\ pancreatitis crónica en un hospital de alta \\ complejidad en Cali, 2011 a 2017. Rev Colomb \\ Gastroenterol. 2021;36(1):30-38. https://doi. \\ org/10.22516/25007440.568 \\ Fundación Valle del Lili, Departamento \\ de Gastroenterología y Hepatología. Cali, \\ Colombia. \\ 2 Fundación Valle del Lili, Centro de \\ Investigaciones Clínicas, Cali, Colombia. \\ Universidad ICESI, Facultad Ciencias de la \\ Salud, Estudiantes de Medicina. Cali, Colombia. \\ Universidad ICESI, Facultad Ciencias de Ia \\ Salud, Departamento de Medicina Interna. Cali, \\ Colombia. \\ *Correspondencia: Carlos A. Rojas-Rodríguez. \\ croj016@yahoo.com
}

Fecha recibido: $\quad 13 / 05 / 20$ Fecha aceptado: $01 / 06 / 20$

\section{Resumen}

Introducción: la pancreatitis crónica (PC) es una inflamación progresiva del páncreas que puede llevar a su destrucción e insuficiencia irreversibles. Es una patología desafiante para el médico, debido a que establecer su diagnóstico puede tomar meses e incluso años, el seguimiento de pacientes suele ser problemático y el conocimiento sobre la clínica y epidemiología en Colombia es incompleto. Este estudio pretende hacer una descripción de pacientes con PC en un centro de referencia en gastroenterología de Cali, Colombia. Metodología: estudio de corte transversal de pacientes adultos con PC confirmada por criterios clínicos y radiológicos entre 2011 y 2017. Resultados: se incluyeron 36 pacientes con PC. La mayoría fueron hombres $(72,2 \%)$ y la media de edad fue $56(+15,1)$ años. El dolor abdominal crónico fue la presentación clínica más común $(83,3 \%)$. Cerca de un cuarto de pacientes presentaba diabetes mellitus $(22,2 \%)$. Se consideró etiología idiopática en $58,3 \%$, alcohólica en el $11,0 \%$ y biliar en el $11,0 \%$. La tomografía axial computarizada (TAC), resonancia magnética (RMN) y colangiopancreatografía por RMN fueron los métodos diagnósticos más usados $(60,5 \%)$, con los que en su mayoría se visualizó atrofia $(53,1 \%)$, dilatación de conductos $(49,0 \%)$ y calcificaciones del páncreas $(34,7 \%)$. Conclusión: los síntomas inespecíficos de la PC en fases iniciales y su largo curso clínico favorecen al subdiagnóstico de esta patología. Los resultados presentados pueden contribuir a la futura creación de escalas clínicas locales que orienten estudios radiológicos y genéticos tempranos, con el fin de lograr un diagnóstico oportuno y mejorar la calidad de vida de estos pacientes.

\section{Palabras clave}

Pancreatitis crónica, epidemiología, dolor abdominal, insuficiencia pancreática exocrina.

\begin{abstract}
Introduction: Chronic pancreatitis $(\mathrm{CP})$ is a progressive inflammation of the pancreas that can lead to irreversible damage and failure. This condition poses great challenges to physicians since its diagnosis can take months or even years. Patient follow-up is often problematic and knowledge about its clinical presentation and epidemiology in Colombia is scarce. This study aims to describe patients with $\mathrm{CP}$ treated at a gastroenterology reference center in Cali, Colombia. Methodology: Cross-sectional study in adult patients with CP confirmed based on clinical and radiological criteria between 2011 and 2017. Results: 36 patients with CP were included. The majority were men $(72.2 \%)$, and the mean age was $56(+15.1)$ years. Chronic abdominal pain was the most common clinical presentation (83.3\%). About a quarter of patients had diabetes mellitus (22.2 \%). Etiology was idiopathic in $58.3 \%$, alcoholic in $11.0 \%$, and biliary in $11.0 \%$. Computed tomography $(\mathrm{CT})$, magnetic resonance imaging (MRI), and MRI cholangiopancreatography were the most commonly used diagnostic methods $(60.5 \%)$, showing mostly atrophy $(53.1 \%)$, duct dilation $(49.0 \%)$, and pancreatic calcifications (34.7\%). Conclusion: Nonspecific symptoms of CP in early stages and its long clinical course favor the underdiagnosis of this condition. The results presented may contribute to the future creation of local clinical scales that guide early radiological and genetic studies to achieve a timely diagnosis and improve the quality of life of these patients.
\end{abstract}

\section{Keywords}

Chronic pancreatitis, Epidemiology, Abdominal pain, Exocrine pancreatic insufficiency. 


\section{INTRODUCCIÓN}

La pancreatitis crónica (PC) fue descrita en 1946 por Comfort y colaboradores (1); sin embargo, su definición, patogénesis y curso clínico continúan siendo debatidos (2), al igual que el consenso sobre técnicas diagnósticas y de manejo (3). Diferentes sociedades científicas han buscado una definición mecanicista y taxonómica desde su descripción inicial, intentando englobar factores de riesgo, etiologías, síntomas y cambios morfológicos del páncreas (4). Se tuvo en cuenta la Sociedad Japonesa $(4,5)$, que define la PC como inflamación, cicatrización y fibrosis progresivas del páncreas que pueden llevar a una pérdida irreversible de su función endocrina y exocrina en personas con predisposición genética, ambiental o de otro tipo (4).

Las características clínicas de PC son inespecíficas al inicio, pero puede presentarse con dispepsia hasta en el $85 \%$, diabetes mellitus (DM) e insuficiencia pancreática exocrina (IPE) (6-10). Una de las principales causas de morbimortalidad es la malabsorción, que genera deficiencia de vitaminas y enfermedad metabólica ósea en estados avanzados de $\operatorname{IPE}(6,7,11,12)$. Un metaanálisis (13) mostró que los pacientes con PC tenían 13 veces más riesgo de desarrollar cáncer de páncreas (14), mientras otros estudios han demostrado mayor mortalidad asociada con la PC al compararla con la población general (15-17).

$\mathrm{El}$ abordaje de un paciente con manifestaciones de PC como dolor abdominal crónico, diarrea o déficit nutricional es reconocer la posibilidad de esta enfermedad, realizar una historia clínica y examen físico dirigidos a condiciones asociadas y aplicar escalas de riesgo, con el fin de buscar cambios sugestivos en laboratorios e imágenes $(7,18)$. Existen condiciones que pueden llevar al desarrollo de PC en lugar de ser provocada por una sola etiología, como el consumo crónico de alcohol, tabaquismo, medicamentos, enfermedades metabólicas tipo hipercalcemia e hipertrigliceridemia, predisposición genética y antecedentes familiares o personales de patología anatómica, litiásica y pancreatitis aguda recurrente $(7,19,20)$.

$\mathrm{Su}$ diagnóstico, además de la presentación clínica, se establece con cambios morfológicos e histológicos del parénquima y conductos pancreáticos (7). La biopsia sigue siendo el estándar de oro; sin embargo, es un método invasivo y difícil de realizar $(7,21)$. El diagnóstico es especialmente importante en fases iniciales para evitar la progresión a la insuficiencia pancreática sintomática e irreversible, por lo que se ha evolucionado con los avances tecnológicos y radiológicos, en busca de un diagnóstico más temprano $(7,18)$. Inicialmente, se usó la radiografía, en la que se visualizan calcificaciones pancreáticas $(3,22)$. Luego, la ecografía, colangiopancreatografía retrógrada endoscópica (CPRE) y tomografía axial computarizada (TAC) emer- gieron como pruebas diagnósticas, debido a que refleja con mayor detalle la anatomía del páncreas (21). Actualmente, la resonancia magnética (RMN) y ultrasonografía endoscópica (USE) son capaces de detectar cambios tempranos y tardíos en el parénquima y los conductos pancreáticos con alta sensibilidad y especificidad para PC (23-25).

El conocimiento actual sobre la epidemiología y las características clínicas locales de la PC está incompleto. El largo curso clínico de la enfermedad, el complejo seguimiento de pacientes (especialmente en consumidores crónicos de alcohol) y las múltiples recaídas y remisiones típicas de estos pacientes hacen que el diagnóstico de PC pueda tomar meses e incluso años (26); por esta razón, permanece subdiagnosticada y los pacientes quedan por fuera del alcance de la gastroenterología. La epidemiología de cada población es fundamental para establecer protocolos clínicos, con el fin de lograr un diagnóstico temprano, atención oportuna y menor carga de la enfermedad. Este estudio pretende hacer una descripción de pacientes con PC en un centro de referencia en gastroenterología del suroccidente colombiano desde 2011 hasta 2017.

\section{METODOLOGÍA}

Se realizó un estudio tipo corte transversal a partir de la búsqueda en historias clínicas de pacientes adultos con PC valorados por consulta externa de gastroenterología. Para la identificación de pacientes se revisaron registros con diagnósticos CIE-10 de PC: K86.0, K86.1, K86.8 y K86.9, entre enero de 2011 y diciembre de 2017. Luego, se confirmó la ocurrencia de la enfermedad al revisar características clínicas y radiológicas de pacientes elegibles.

Se incluyeron pacientes mayores de 18 años, de ambos sexos, con dolor abdominal crónico, DM o esteatorrea, y características radiológicas que incluían cálculos en la vesícula o la vía biliar, cambios en el parénquima del páncreas como atrofia, calcificaciones, quistes, lobularidad, cálculos o dilatación del conducto pancreático evidentes en la radiografía, ecografía, TAC, RMN, colangiopancreatografía por resonancia magnética (CPRM) o USE. Se excluyeron pacientes con imágenes que reportaban páncreas normal.

La etiología de la PC fue establecida por el especialista tratante dependiendo de factores de riesgo y hallazgos anormales en estudios de laboratorio y radiológicos.

Para el análisis de datos se usó Stata v.14 (StataCorp. 2015. Stata Statistical Software: Ralease 14. College Station, TX: StataCorp LP). La distribución de variables cuantitativas fue evaluada con la prueba de Shapiro-Wilk, y se utilizaba la media o mediana con su respectiva desviación estándar (DE) o rango intercuartílico. Se realizaron proporciones para variables cualitativas. Los resultados se muestran en tablas de frecuencia y gráficos de barras. 
Este proyecto fue aprobado por el comité de ética de la Fundación Valle del Lili con acta n. ${ }^{\circ} 09$ del 9 de mayo del 2018. Todos los investigadores manifestaron no tener conflictos de interés.

\section{RESULTADOS}

Se identificaron inicialmente 97 pacientes con PC según los diagnósticos CIE-10 mencionados dentro del período establecido para el estudio. Al revisar los criterios de selección, 36 pacientes cumplieron y fueron incluidos en el análisis. La razón principal de exclusión fue no contar con el reporte del estudio radiológico en los registros médicos.

Las características demográficas y antecedentes médicos de la población se muestran en la Tabla 1. La mayoría de los pacientes con PC fueron hombres $(72,2 \%)$ y la media de edad fue de $56 \pm 15,1$ años. Los antecedentes médicos más frecuentes fueron hipertensión arterial (HTA) (38,9\%), consumo crónico de alcohol (25,0 \%) y DM (22,2 \%). En 3 pacientes se identificaron antecedentes familiares en primer grado, 2 tenían PC (5,6\%) y 1 tenía cáncer de páncreas (2,8\%).

Tabla 1. Características demográficas y antecedentes médicos de la población con $\mathrm{PC}^{*}$

\begin{tabular}{|c|c|}
\hline & $\mathrm{n}(\%)$ \\
\hline Edad $^{* *}$ & $56 \pm 15,1$ \\
\hline \multicolumn{2}{|l|}{ Sexo } \\
\hline - Masculino & $26(72,2)$ \\
\hline - Femenino & $10(27,8)$ \\
\hline \multicolumn{2}{|l|}{ Antecedentes personales } \\
\hline - DM & $8(22,2)$ \\
\hline - Insulinoterapia & $5(13,9)$ \\
\hline - HTA & $14(38,9)$ \\
\hline - Dislipidemia & $5(13,9)$ \\
\hline - Consumo crónico de alcohol & $9(25,0)$ \\
\hline - Tabaquismo & $4(11,1)$ \\
\hline - Hipercalcemia & 0 \\
\hline - Insuficiencia renal crónica & $3(8,3)$ \\
\hline - Pancreatitis aguda necrotizante & $2(5,6)$ \\
\hline - Cáncer de páncreas & $1(2,8)$ \\
\hline \multicolumn{2}{|l|}{ Antecedentes familiares } \\
\hline - Cáncer de páncreas & $1(2,8)$ \\
\hline$-P C$ & $2(5,6)$ \\
\hline
\end{tabular}

${ }^{*} \mathrm{n}=36 \cdot{ }^{* *}$ Valor expresado en media $( \pm \mathrm{DE})$.
La forma de presentación clínica más común fue dolor abdominal crónico (83,3\%) (Tabla 2). Otros síntomas menos frecuentes fueron la pérdida de peso $(30,6 \%)$, diarrea crónica $(27,8 \%)$ y náuseas o vómito (19,4\%). 8 de 36 pacientes $(22,2 \%)$ presentaban DM.

Tabla 2. Presentación clínica de pacientes con PC*

\section{Síntoma}

n (\%)

Dolor abdominal crónico $30(83,3)$

Pérdida de peso $11(30,6)$

Diarrea crónica $10(27,8)$

DM $8(22,2)$

Ictericia $3(8,3)$

Náuseas y vómito $7(19,4)$

Distensión abdominal $5(13,9)$

Dispepsia

$5(13,9)$

Estreñimiento $3(8,3)$

Hiporexia $2(5,6)$

Coluria $1(2,8)$

${ }^{*} \mathrm{n}=36$.

Para más de la mitad de pacientes (58,3\%) se determinó que la PC tuvo un origen idiopático (Figura 1). Otras etiologías identificadas fueron la patología biliar $(11,0 \%)$ y el consumo crónico de alcohol (11,0\%). En cuanto al estudio radiológico, se realizaron 81 estudios en los 36 pacientes (Tabla 3). La TAC, RMN y CPRM fueron los métodos diagnósticos realizados en cerca de dos tercios de pacientes $(60,5 \%)$ y sus principales hallazgos fueron atrofia $(53,1 \%)$, calcificaciones $(34,7 \%)$ y dilatación del conducto pancreático (49,0\%). Se usó USE en el 14,8 \% de pacientes.

\section{DISCUSIÓN}

La PC es una inflamación crónica del páncreas y su insuficiencia era necesaria para el diagnóstico, según ciertas sociedades científicas; esto condicionaba al reconocimiento tardío de la enfermedad $(4,7)$. Por esta razón, se ha visto la necesidad de incluir en la definición los factores de riesgo y condiciones asociadas que permitan reconocerla de manera oportuna, para impedir el retraso en su diagnóstico (7). El abordaje de la PC requiere un amplio margen de sospecha clínica. Se encontró que la mayoría de pacientes eran hombres y consultaban por dolor abdominal, pérdida 


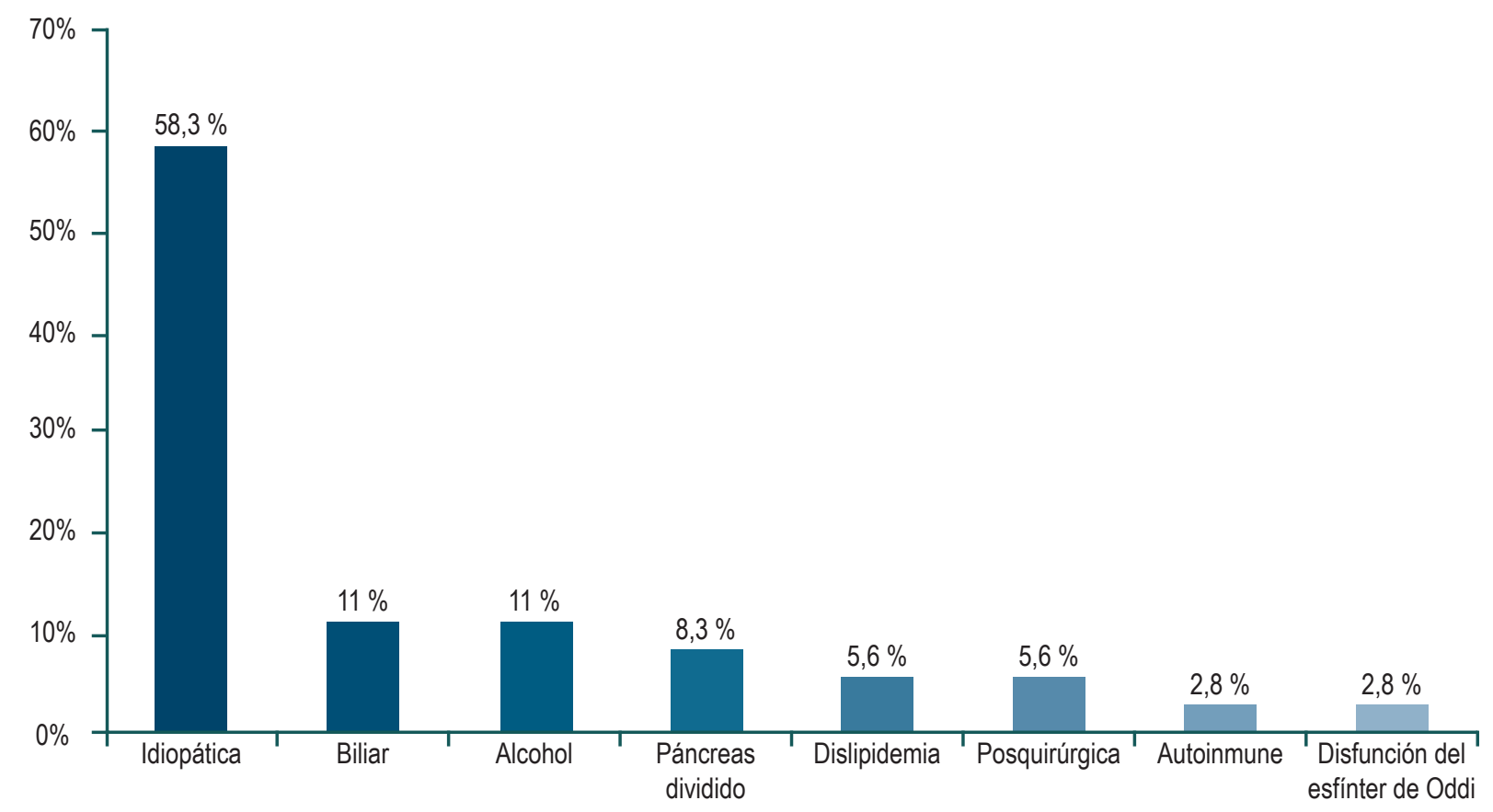

Figura 1. Etiologías identificadas en los pacientes con PC.

de peso y diarrea crónicos, y estos también son indicativos de IPE. Una proporción considerable de pacientes era diabética y mostraba atrofia pancreática, lo que contribuye a pensar que se encontraban en estados avanzados de la enfermedad con páncreas deteriorado, tanto en su función exocrina como endocrina, y plantea que el tiempo de remisión a gastroenterología es tardío. Con esta información se pretende complementar el conocimiento de médicos que atienden poblaciones similares, en busca de la sospecha y reconocimiento precoz, con el fin de lograr un diagnóstico oportuno con la mejoría de la atención de los pacientes.

Este estudio muestra características demográficas similares a las reportadas en registros internacionales $(1,17,27$, 28). Entre los antecedentes más frecuentes se encuentran la HTA y dislipidemia, los cuales también son considerados factores de riesgo cardiovascular. Esto es esperable, puesto que en pacientes con PC y especialmente con IPE, se ha demostrado mayor riesgo de enfermedades cardiovasculares como insuficiencia cardíaca (29), infarto agudo de miocardio (30) y HTA (31). Otros antecedentes médicos como la hipertrigliceridemia se han relacionado con PC $(7,20,32)$. El NASP group de Estados Unidos identificó la hipertrigliceridemia como un factor de riesgo en el $13 \%$ de pacientes (33), lo que puede explicar la frecuencia encontrada en la población. A pesar de reconocer antecedentes familiares de enfermedad pancreática como factores de riesgo para PC $(20,34)$, en nuestra población fueron poco prevalentes, probablemente por desconocimiento de los pacientes y por falta de estudios genéticos disponibles.

La PC se presenta con dolor abdominal crónico hasta en el $85 \%$ de pacientes (1), similar a la frecuencia encontrada en este estudio. El dolor es típicamente en el epigastrio, no mejora con antiácidos y altera significativamente la calidad de vida (8-10). Puede ser fácilmente interpretado como un trastorno funcional tipo síndrome de intestino irritable o dispepsia. Este grupo de pacientes debería tener una observación especial, en la que es fundamental la caracterización semiológica del dolor, el estudio y seguimiento, que le permita al clínico sospechar o desestimar una posible PC.

Entre el $41 \%$ y el $86 \%$ de pacientes con PC desarrollan DMa lolargo de su vida (11). Llamala atención que el $22,2 \%$ de la población ya presentaba DM y es un dato significativo debido al tamaño de muestra y la media de edad. Otras manifestaciones clínicas de IPE, como mal digestión, pueden provocar diarrea crónica o esteatorrea en $22,5 \%$ (35) y pérdida de peso (36), características más frecuentes en esta investigación después del dolor. Los síntomas de PC en fases iniciales pueden ser inespecíficos como náuseas, vómito, estreñimiento y distensión abdominal (22), presentes en cerca de dos tercios de pacientes en este estudio. $\mathrm{Su}$ presentación aislada puede deberse a diferentes patologías, situación que retrasaría el diagnóstico de PC. 
Tabla 3. Estudios radiológicos usados para el diagnóstico de PC*

\begin{tabular}{|c|c|}
\hline Estudio & n (\%) \\
\hline Radiografía de abdomen & $2(2,5)$ \\
\hline - Calcificaciones en páncreas & $2(100)$ \\
\hline Ecografía de abdomen & $18(22,2)$ \\
\hline - Cálculos en la vesícula o la vía biliar & $6(33,3)$ \\
\hline - Atrofia pancreática & $4(22,2)$ \\
\hline - Calcificaciones en el páncreas & $2(11,1)$ \\
\hline - Cálculos en el conducto pancreático & $5(27,8)$ \\
\hline - Dilatación del conducto pancreático & $5(27,8)$ \\
\hline RMN o CPRM & $24(29,6)$ \\
\hline - Cálculos en la vía biliar & $4(16,7)$ \\
\hline - Atrofia pancreática & $14(58,3)$ \\
\hline - Calcificaciones en el páncreas & $5(20,8)$ \\
\hline - Cálculos en el conducto pancreático & $8(33,3)$ \\
\hline - Dilatación del conducto pancreático & $14(58,3)$ \\
\hline - Quistes pancreáticos & $3(12,5)$ \\
\hline - Lobularidad pancreática & $3(12,5)$ \\
\hline TAC de abdomen & $25(30,9)$ \\
\hline - Atrofia pancreática & $12(48,0)$ \\
\hline - Calcificaciones en el páncreas & $12(48,0)$ \\
\hline - Cálculos en el conducto pancreático & $4(16,0)$ \\
\hline - Dilatación del conducto pancreático & $10(40,0)$ \\
\hline - Quistes pancreáticos & $3(12,0)$ \\
\hline USE & $12(14,8)$ \\
\hline - Cálculos en la vesícula o la vía biliar & $2(16,7)$ \\
\hline - Atrofia pancreática & $5(41,7)$ \\
\hline - Calcificaciones en el páncreas & $8(66,7)$ \\
\hline - Cálculos en el conducto pancreático & $6(50,0)$ \\
\hline - Dilatación del conducto pancreático & $12(100)$ \\
\hline - Quistes pancreáticos & $3(25,0)$ \\
\hline - Lobularidad pancreática & $6(50,0)$ \\
\hline Criterios de Rosemont en USE & $12(14,8)$ \\
\hline - Diagnóstico de PC & $4(33,3)$ \\
\hline - Sugestivo de PC & $7(58,3)$ \\
\hline - Indeterminado para PC & $1(8,3)$ \\
\hline
\end{tabular}

En relación con la etiología, se reportó PC idiopática en más de la mitad de la población. En China en 2018 se realizó un censo con 2037 pacientes, de los cuales el 80,2 \% de los casos fue por etiología idiopática y $19,8 \%$ por etiología alcohólica (35). Estos resultados coinciden con los hallazgos de este estudio. Un cuarto de los pacientes reportó consumo crónico de alcohol, pero solo el 11,0 \% fue considerado de origen alcohólico, esto hace pensar que el alcohol es un factor contribuyente, pero no exclusivo para PC. Las guías (7) recomiendan tener en cuenta escalas como TIGAR-O (20) y M-ANNHEIM (34), las cuales describen factores de riesgo y etiologías. La PC se desarrolla a partir de la interacción de estos factores $(7,19)$ $y$ es individual y diferente en cada paciente. Las etiologías más frecuentes en series mundiales con rangos amplios son la alcohólica e idiopática $(1,27,33,35,37,38)$. Estudios recientes han considerado el consumo de alcohol y tabaco como los factores más influyentes para PC $(1,19,27,39$, $40)$. Esto coincide con el estudio de Wilcox y colaboradores (38), en el que el origen alcohólico fue de $41,9 \%$, el idiopático fue de $27,8 \%$ y el genético fue de $10,2 \%$. Sin embargo, estos resultados varían ampliamente según los hábitos y estilo de vida de cada población estudiada.

De aquellos pacientes clasificados como PC idiopática, actualmente se conoce que hasta el $50 \%$ presenta mutaciones del gen inhibidor de la tripsina (SPINK1) o del gen regulador de la conductancia transmembrana de la fibrosis quística (CFTR) y, aproximadamente, el $1 \%$ puede tener pancreatitis hereditaria asociada con mutaciones genéticas del tripsinógeno catiónico (PRSS1) (41-44). Las mutaciones descritas predisponen a un estado de inflamación crónica que genera la fibrosis del páncreas $(41,45,46)$. La presencia de estas mutaciones no fue evaluada en este estudio posiblemente debido a su alto costo y falta de disponibilidad, situación que es frecuente en muchos centros y contribuye al subdiagnóstico de esta etiología.

Las pruebas radiológicas son sensibles en presencia de enfermedad avanzada, pero limitadas para diagnosticar etapas tempranas de PC $(7,8,18)$. La TAC, RMN y CPRM fueron los métodos diagnósticos más usados en este estudio, lo que coincide con las recomendaciones de las últimas guías del AGC (7). La visualización de atrofia (53,1 \%), calcificaciones y dilatación de conductos pancreáticos $(49,0 \%)$ con estos métodos es similar a la encontrada en estudios internacionales $(54,0 \%$ y $68,0 \%$, respectivamente) $(18,47)$. La TAC tiene una sensibilidad del $75 \%$ para el diagnóstico de PC (intervalo de confianza [IC] del 95 \%: 66 \%-83 \%) (7, 8). En comparación con la RMN y CPRM, su costo y disponibilidad hacen que la TAC sea la prueba de preferencia en pacientes con sospecha de PC.

La RMN tiene una sensibilidad del 78 \% (IC $95 \%$ : $69 \%$ $85 \%$ ) (7). Puede proporcionar imágenes de alta calidad 
tanto del parénquima pancreático como del sistema ductal. Recientemente, se ha usado la administración intravenosa de secretina durante la CPRM, pues permite ver el mayor número de ramas ductales y el llenado duodenal, el cual se considera un marcador dinámico de la función pancreática exocrina (8). Según las guías recientes, si la TAC muestra resultados normales, pero la sospecha de PC es alta, se debe obtener una CPRM para evaluar los cambios ductales (48).

La USE también está indicada cuando los resultados de la TAC, RMN o CPRM son normales, pero existe alta sospecha clínica de PC (7). Se usó USE en el 14,8 \% de pacientes en este estudio, todos presentaban dilatación del conducto pancreático y se reportó en la mayoría diagnóstico sugestivo, según la clasificación de Rosemont (49). Este sistema incluye 5 criterios parenquimatosos y 5 ductales, se clasifica la PC en diagnóstico, sugestivo, indeterminado o normal (49). A pesar de que su sensibilidad es la más alta en comparación con los demás estudios (81 \%, IC $95 \%$ : 70 \%-89\%), es un método invasivo, no se encuentra fácilmente disponible y puede haber diferencias interobservador (7).

La fortaleza de este estudio es poner en evidencia las manifestaciones clínicas con las que se presentan pacientes con PC a la consulta externa de gastroenterología a nivel local: con enfermedad avanzada, caracterizada tanto por hallazgos radiológicos, comorbilidades y síntomas. Este conocimiento podrá ser utilizado en la valoración de los pacientes, con el fin de establecer protocolos clínicos optimizando recursos para un diagnóstico temprano y una menor carga de la enfermedad. Como limitaciones se encuentra la naturaleza retrospectiva del estudio, que impidió realizar una evaluación completa del paciente en tiempo real y llevó a la exclusión de muchos participantes. No se realizaron pruebas genéticas por el costo y falta de disponibilidad. Esta es una brecha para fortalecer en el futuro mientras se globalizan avances tecnológicos y nuevos métodos diagnósticos que logren una evaluación más completa de estos pacientes.

\section{CONCLUSIÓN}

Las características demográficas de nuestra población son similares a las observadas en estudios internacionales. La PC se presenta con síntomas inespecíficos iniciales, que se pueden confundir con trastornos funcionales o, de manera más frecuente, en estados avanzados de la enfermedad como IPE y DM, por lo que su diagnóstico es un reto en la práctica clínica. En ocasiones, los factores de riesgo no son fáciles de demostrar y el estudio radiológico adecuado no siempre se encuentra disponible, lo que favorece aún más al subdiagnóstico de esta patología. Dada la alta frecuencia de PC idiopática, son necesarios estudios prospectivos en los que se busquen activamente factores de riesgo y se logren determinar variantes genéticas, especialmente en pacientes jóvenes sin causa evidente. Los resultados presentados pueden contribuir a la creación de escalas clínicas locales que orienten estudios tempranos, con el fin de clasificar de manera correcta y oportuna la PC, para mejorar el pronóstico y la calidad de vida de nuestros pacientes.

\section{REFERENCIAS}

1. Muniraj T, Aslanian HR, Farrell J, Jamidar PA. Chronic pancreatitis, a comprehensive review and update. Part I: epidemiology, etiology, risk factors, genetics, pathophysiology, and clinical features. Dis Mon. 2014;60(12):530-50. https://doi.org/10.1016/j.disamonth.2014.11.002

2. Steer ML, Waxman I, Freedman S. Chronic pancreatitis. N Engl J Med. 1995;332(22):1482-90. https://doi. org/10.1056/NEJM199506013322206

3. Arango M LA, Díaz T CP, Caicedo QCA, Ángel Rodríguez C. Estado actual del diagnóstico y manejo de la pancreatitis crónica. Revista Colombiana de Gastroenterologia. 2019;34(4):376-84. https://doi. org/10.22516/25007440.301

4. Whitcomb DC, Frulloni L, Garg P, Greer JB, Schneider A, Yadav D, Shimosegawa T. Chronic pancreatitis: An international draft consensus proposal for a new mechanistic definition. Pancreatology. 2016;16(2):218-24. https://doi. org/10.1016/j.pan.2016.02.001
5. Shimosegawa T, Kataoka K, Kamisawa T, Miyakawa H, Ohara H, Ito T, Naruse S, Sata N, Suda K, Hirota M, Takeyama Y, Shiratori K, Hatori T, Otsuki M, Atomi Y, Sugano K, Tanaka M. The revised Japanese clinical diagnostic criteria for chronic pancreatitis. J Gastroenterol. 2010;45(6):584-91. https://doi.org/10.1007/s00535010-0242-4

6. Layer P, Yamamoto H, Kalthoff L, Clain JE, Bakken LJ, DiMagno EP. The different courses of early-and lateonset idiopathic and alcoholic chronic pancreatitis. Gastroenterology. 1994;107(5):1481-7. https://doi. org/10.1016/0016-5085(94)90553-3

7. Gardner TB, Adler DG, Forsmark CE, Sauer BG, Taylor JR, Whitcomb DC. ACG Clinical Guideline: Chronic Pancreatitis. Am J Gastroenterol. 2020;115(3):322-339. https://doi.org/10.14309/ajg.0000000000000535

8. Singh VK, Yadav D, Garg PK. Diagnosis and Management of Chronic Pancreatitis: A Review. JAMA. 
2019;322(24):2422-34. https://doi.org/10.1001/ jama.2019.19411

9. Mullady DK, Yadav D, Amann ST, O’Connell MR, Barmada MM, Elta GH, Scheiman JM, Wamsteker EJ, Chey WD, Korneffel ML, Weinman BM, Slivka A, Sherman S, Hawes RH, Brand RE, Burton FR, Lewis MD, Gardner TB, Gelrud A, DiSario J, Baillie J, Banks PA, Whitcomb DC, Anderson MA; NAPS2 Consortium. Type of pain, pain-associated complications, quality of life, disability and resource utilisation in chronic pancreatitis: a prospective cohort study. Gut. 2011;60(1):77-84. https://doi. org/10.1136/gut.2010.213835

10. Wilcox CM, Yadav D, Ye T, Gardner TB, Gelrud A, Sandhu BS, Lewis MD, Al-Kaade S, Cote GA, Forsmark CE, Guda NM, Conwell DL, Banks PA, Muniraj T, Romagnuolo J, Brand RE, Slivka A, Sherman S, Wisniewski SR, Whitcomb DC, Anderson MA. Chronic pancreatitis pain pattern and severity are independent of abdominal imaging findings. Clin Gastroenterol Hepatol. 2015 Mar;13(3):552-60; quiz e28-9. https://doi.org/ 10.1016/j.cgh.2014.10.015

11. Malka D, Hammel P, Sauvanet A, Rufat P, O’Toole D, Bardet P, Belghiti J, Bernades P, Ruszniewski P, Lévy P. Risk factors for diabetes mellitus in chronic pancreatitis. Gastroenterology. 2000;119(5):1324-32. https://doi. org/10.1053/gast.2000.19286

12. Lankisch PG, Löhr-Happe A, Otto J, Creutzfeldt W. Natural course in chronic pancreatitis. Pain, exocrine and endocrine pancreatic insufficiency and prognosis of the disease. Digestion. 1993;54(3):148-55. https://doi. org/10.1159/000201029

13. Raimondi S, Lowenfels AB, Morselli-Labate AM, Maisonneuve P, Pezzilli R. Pancreatic cancer in chronic pancreatitis; aetiology, incidence, and early detection. Best Pract Res Clin Gastroenterol. 2010;24(3):349-58. https:// doi.org/10.1016/j.bpg.2010.02.007

14. Lowenfels AB, Maisonneuve P, DiMagno EP, Elitsur Y, Gates LK, Perrault J, Whitcomb DC. Hereditary pancreatitis and the risk of pancreatic cancer. International Hereditary Pancreatitis Study Group. J Natl Cancer Inst. 1997;89(6):442-6. https://doi.org/10.1093/jnci/89.6.442

15. Nøjgaard C, Bendtsen F, Becker U, Andersen JR, Holst C, Matzen P. Danish patients with chronic pancreatitis have a four-fold higher mortality rate than the Danish population. Clinical Gastroenterology and Hepatology. 2010;8(4):38490. https://doi.org/10.1016/j.cgh.2009.12.016

16. Levy P, Milan C, Pignon JP, Baetz A, Bernades P. Mortality factors associated with chronic pancreatitis. Unidimensional and multidimensional analysis of a medical-surgical series of 240 patients. Gastroenterology. 1989;96(4):1165-72. https://doi.org/10.1016/00165085(89)91637-5

17. Yadav D, Timmons L, Benson JT, Dierkhising RA, Chari ST. Incidence, prevalence, and survival of chronic pancreatitis: a population-based study. Am J Gastroenterol. 2011;106(12):2192-9. https://doi.org/10.1038/ ajg. 2011.328
18. Conwell DL, Lee LS, Yadav D, Longnecker DS, Miller FH, Mortele KJ, Levy MJ, Kwon R, Lieb JG, Stevens T, Toskes PP, Gardner TB, Gelrud A, Wu BU, Forsmark CE, Vege SS. American Pancreatic Association Practice Guidelines in Chronic Pancreatitis: evidence-based report on diagnostic guidelines. Pancreas. 2014;43(8):1143-62. https://doi. org/10.1097/MPA.0000000000000237

19. Yadav D, Whitcomb DC. The role of alcohol and smoking in pancreatitis. Nat Rev Gastroenterol Hepatol. 2010;7(3):131-45. https://doi.org/10.1038/nrgastro.2010.6

20. Whitcomb DC, Group NAPS. Pancreatitis: TIGAR-O Version 2 Risk/Etiology Checklist With Topic Reviews, Updates, and Use Primers. Clin Transl Gastroenterol. 2019;10(6):e00027. https://doi.org/10.14309/ ctg.0000000000000027

21. Etemad B, Whitcomb DC. Chronic pancreatitis: diagnosis, classification, and new genetic developments. Gastroenterology. 2001;120(3):682-707. https://doi. org/10.1053/gast.2001.22586

22. Czul F, Coronel E, Donet JA. Una actualización de pancreatitis crónica: artículo de revisión. Rev Gastroenterol Perú. 2017;37(2):146-55.

23. Seicean A. Endoscopic ultrasound in chronic pancreatitis: where are we now? World J Gastroenterol. 2010;16(34):4253-63. https://doi.org/10.3748/wjg.v16. i34.4253

24. Issa Y, Kempeneers MA, van Santvoort HC, Bollen TL, Bipat S, Boermeester MA. Diagnostic performance of imaging modalities in chronic pancreatitis: a systematic review and meta-analysis. Eur Radiol. 2017;27(9):3820-44. https://doi.org/10.1007/s00330-016-4720-9

25. Sahai AV, Mishra G, Penman ID, Williams D, Wallace MB, Hadzijahic N, Pearson A, Vanvelse A, Hoffman BJ, Hawes RH. EUS to detect evidence of pancreatic disease in patients with persistent or nonspecific dyspepsia. Gastrointest Endosc. 2000;52(2):153-9. https://doi. org/10.1067/mge.2000.107910

26. Lévy P, Domínguez-Muñoz E, Imrie C, Löhr M, Maisonneuve P. Epidemiology of chronic pancreatitis: burden of the disease and consequences. United European Gastroenterol J. 2014;2(5):345-54. https://doi. org/10.1177/2050640614548208

27. Hirota M, Shimosegawa T, Masamune A, Kikuta K, Kume K, Hamada S, Kanno A, Kimura K, Tsuji I, Kuriyama S; Research Committee of Intractable Pancreatic Diseases. The seventh nationwide epidemiological survey for chronic pancreatitis in Japan: clinical significance of smoking habit in Japanese patients. Pancreatology. 2014;14(6):490-6. https://doi.org/10.1016/j.pan.2014.08.008

28. Lin Y, Tamakoshi A, Matsuno S, Takeda K, Hayakawa T, Kitagawa M, et al. Nationwide epidemiological survey of chronic pancreatitis in Japan. J Gastroenterol. 2000;35(2):136-41. https://doi.org/10.1007/ s005350050026 
29. Nikolic S, Dugic A, Steiner C, Tsolakis AV, Haugen Löfman IM, Löhr JM, Vujasinovic M. Chronic pancreatitis and the heart disease: Still terra incognita? World J Gastroenterol. 2019;25(44):6561-70. https://doi.org/10.3748/wjg.v25. i44.6561

30. Hsu MT, Lin CL, Chung WS. Increased Risk of Acute Coronary Syndrome in Patients With Chronic Pancreatitis: A Nationwide Cohort Analysis. Medicine (Baltimore). 2016;95(20):e3451. https://doi.org/10.1097/ MD.0000000000003451

31. Gullo L, Stella A, Labriola E, Costa PL, Descovich G, Labò G. Cardiovascular lesions in chronic pancreatitis: a prospective study. Dig Dis Sci. 1982;27(8):716-22. https://doi. org/10.1007/BF01393767

32. Yadav D, Lowenfels AB. The epidemiology of pancreatitis and pancreatic cancer. Gastroenterology. 2013;144(6):1252-61. https://doi.org/10.1053/j.gastro.2013.01.068

33. Conwell DL, Banks PA, Sandhu BS, Sherman S, Al-Kaade $\mathrm{S}$, Gardner TB, Anderson MA, Wilcox CM, Lewis MD, Muniraj T, Forsmark CE, Cote GA, Guda NM, Tian Y, Romagnuolo J, Wisniewski SR, Brand R, Gelrud A, Slivka A, Whitcomb DC, Yadav D. Validation of Demographics, Etiology, and Risk Factors for Chronic Pancreatitis in the USA: A Report of the North American Pancreas Study (NAPS) Group. Dig Dis Sci. 2017;62(8):2133-40. https:// doi.org/10.1007/s10620-017-4621-z

34. Schneider A, Löhr JM, Singer MV. The M-ANNHEIM classification of chronic pancreatitis: introduction of a unifying classification system based on a review of previous classifications of the disease. J Gastroenterol. 2007;42(2):101-19. https://doi.org/10.1007/s00535-006-1945-4

35. Hao L, Wang LS, Liu Y, Wang T, Guo HL, Pan J, Wang D, Bi YW, Ji JT, Xin L, Du TT, Lin JH, Zhang D, Zeng XP, Zou WB, Chen H, Xie T, Li BR, Liao Z, Cong ZJ, Xu $\mathrm{ZL}, \mathrm{Li} \mathrm{ZS}, \mathrm{Hu} \mathrm{LH}$. The different course of alcoholic and idiopathic chronic pancreatitis: A long-term study of 2,037 patients. PLoS One. 2018;13(6):e0198365. https://doi. org/10.1371/journal.pone.0198365

36. Li BR, Pan J, Du TT, Liao Z, Ye B, Zou WB, Chen H, Ji JT, Zheng ZH, Wang D, Lin JH, Ning SB, Hu LH, Li ZS. Risk Factors for Steatorrhea in Chronic Pancreatitis: A Cohort of 2,153 Patients. Sci Rep. 2016;6:21381. https://doi. org/10.1038/srep21381

37. Hirota M, Shimosegawa T, Masamune A, Kikuta K, Kume K, Hamada S, Kihara Y, Satoh A, Kimura K, Tsuji I, Kuriyama S; Research Committee of Intractable Pancreatic Diseases. The sixth nationwide epidemiological survey of chronic pancreatitis in Japan. Pancreatology. 2012;12(2):79-84. https://doi.org/10.1016/j. pan.2012.02.005

38. Wilcox CM, Sandhu BS, Singh V, Gelrud A, Abberbock JN, Sherman S, Cote GA, Al-Kaade S, Anderson MA, Gardner TB, Lewis MD, Forsmark CE, Guda NM, Romagnuolo J, Baillie J, Amann ST, Muniraj T, Tang G, Conwell DL, Banks PA, Brand RE, Slivka A, Whitcomb D, Yadav D.
Racial Differences in the Clinical Profile, Causes, and Outcome of Chronic Pancreatitis. Am J Gastroenterol. 2016;111(10):1488-96. https://doi.org/10.1038/ ajg.2016.316

39. Whitcomb DC, Yadav D, Adam S, Hawes RH, Brand RE, Anderson MA, Money ME, Banks PA, Bishop MD, Baillie J, Sherman S, DiSario J, Burton FR, Gardner TB, Amann ST, Gelrud A, Lo SK, DeMeo MT, Steinberg WM, Kochman ML, Etemad B, Forsmark CE, Elinoff B, Greer JB, O’Connell M, Lamb J, Barmada MM; North American Pancreatic Study Group. Multicenter approach to recurrent acute and chronic pancreatitis in the United States: the North American Pancreatitis Study 2 (NAPS2). Pancreatology. 2008;8(4-5):520-31. https://doi. org/10.1159/000152001

40. Yadav D, Hawes RH, Brand RE, Anderson MA, Money ME, Banks PA, Bishop MD, Baillie J, Sherman S, DiSario J, Burton FR, Gardner TB, Amann ST, Gelrud A, Lawrence C, Elinoff B, Greer JB, O'Connell M, Barmada MM, Slivka A, Whitcomb DC; North American Pancreatic Study Group. Alcohol consumption, cigarette smoking, and the risk of recurrent acute and chronic pancreatitis. Arch Intern Med. 2009;169(11):1035-45. https://doi.org/10.1001/ archinternmed.2009.125

41. Midha S, Khajuria R, Shastri S, Kabra M, Garg PK. Idiopathic chronic pancreatitis in India: phenotypic characterisation and strong genetic susceptibility due to SPINK1 and CFTR gene mutations. Gut. 2010;59(6):800-7. https://doi.org/10.1136/gut.2009.191239

42. Rosendahl J, Landt O, Bernadova J, Kovacs P, Teich N, Bödeker H, Keim V, Ruffert C, Mössner J, Kage A, Stumvoll M, Groneberg D, Krüger R, Luck W, Treiber M, Becker M, Witt H. CFTR, SPINK1, CTRC and PRSS1 variants in chronic pancreatitis: is the role of mutated CFTR overestimated? Gut. 2013;62(4):582-92. https:// doi.org/10.1136/gutjnl-2011-300645

43. Zou WB, Tang XY, Zhou DZ, Qian YY, Hu LH, Yu FF, Yu D, Wu H, Deng SJ, Lin JH, Zhao AJ, Zhao ZH, Wu HY, Zhu JH, Qian W, Wang L, Xin L, Wang MJ, Wang LJ, Fang X, He L, Masson E, Cooper DN, Férec C, Li ZS, Chen JM, Liao Z. SPINK1, PRSS1, CTRC, and CFTR Genotypes Influence Disease Onset and Clinical Outcomes in Chronic Pancreatitis. Clin Transl Gastroenterol. 2018;9(11):204. https://doi.org/10.1038/s41424-018-0069-5

44. Pfützer RH, Barmada MM, Brunskill AP, Finch R, Hart PS, Neoptolemos J, Furey WF, Whitcomb DC. SPINK1/ PSTI polymorphisms act as disease modifiers in familial and idiopathic chronic pancreatitis. Gastroenterology. 2000;119(3):615-23. https://doi.org/10.1053/ gast.2000.18017

45. Rosendahl J, Witt H, Szmola R, Bhatia E, Ozsvári B, Landt O, Schulz HU, Gress TM, Pfützer R, Löhr M, Kovacs P, Blüher M, Stumvoll M, Choudhuri G, Hegyi P, te Morsche RH, Drenth JP, Truninger K, Macek M Jr, Puhl G, Witt U, Schmidt H, Büning C, Ockenga J, Kage A, Groneberg DA, Nickel R, Berg T, Wiedenmann B, Bödeker H, Keim 
V, Mössner J, Teich N, Sahin-Tóth M. Chymotrypsin $\mathrm{C}$ (CTRC) variants that diminish activity or secretion are associated with chronic pancreatitis. Nat Genet. 2008;40(1):78-82. https://doi.org/10.1038/ng.2007.44

46. Whitcomb DC, Gorry MC, Preston RA, Furey W, Sossenheimer MJ, Ulrich CD, Martin SP, Gates LKJr, Amann ST, Toskes PP, Liddle R, McGrath K, Uomo G, Post JC, Ehrlich GD. Hereditary pancreatitis is caused by a mutation in the cationic trypsinogen gene. Nat Genet. 1996;14(2):141-5. https://doi.org/10.1038/ng1096-141

47. Luetmer PH, Stephens DH, Ward EM. Chronic pancreatitis: reassessment with current CT. Radiology. 1989;171(2):353-7. https://doi.org/10.1148/radiology.171.2.2704799
48. Joergensen MT, Brusgaard K, Crüger DG, Gerdes AM, Schaffalitzky de Muckadell OB. Genetic, epidemiological, and clinical aspects of hereditary pancreatitis: a populationbased cohort study in Denmark. Am J Gastroenterol. 2010;105(8):1876-83. https://doi.org/10.1038/ ajg.2010.193

49. Catalano MF, Sahai A, Levy M, Romagnuolo J, Wiersema M, Brugge W, Freeman M, Yamao K, Canto M, Hernandez LV. EUS-based criteria for the diagnosis of chronic pancreatitis: the Rosemont classification. Gastrointest Endosc. 2009;69(7):1251-61. https://doi.org/10.1016/j. gie.2008.07.043 